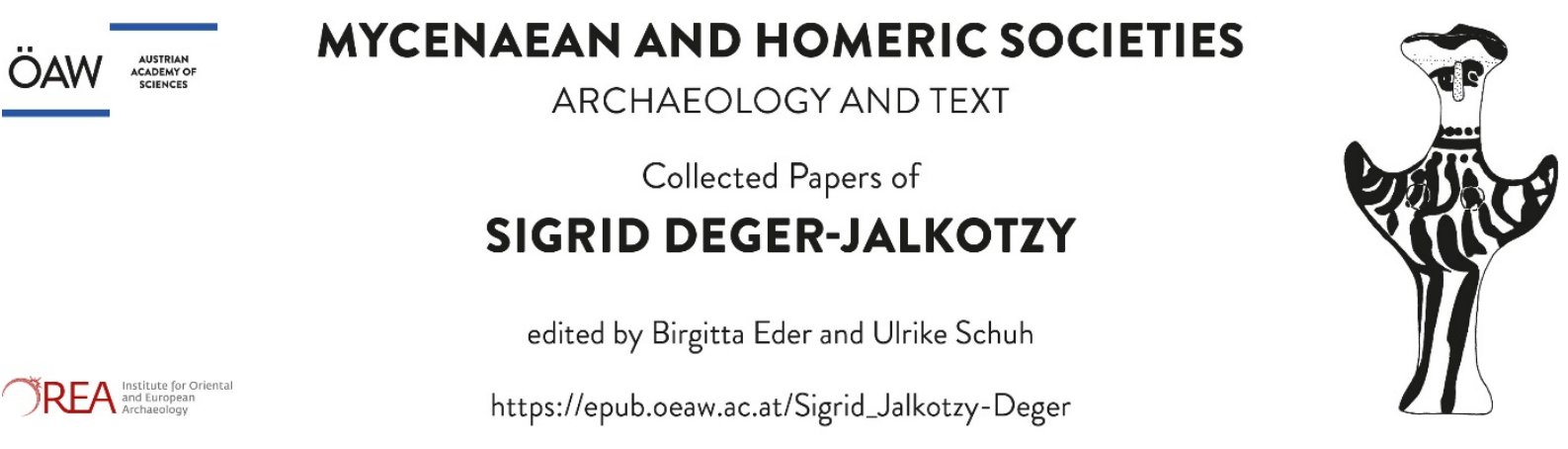

77.

S. Deger-Jalkotzy

Was geschah in Aigina während und nach der mykenischen Palastzeit?

in: C. Reinholdt - P. Scherrer - W. Wohlmayr (eds.), Aiakeion. Beiträge zur Klassischen Altertumswissenschaft zu Ehren von Florens Felten (Vienna 2009) 49-58

(C) Phoibos Verlag

mit freundlicher Genehmigung / with kind permission

Dieses Dokument darf ausschließlich für wissenschaftliche Zwecke genutzt werden (Lizenz CC BY-NC-ND), gewerbliche Nutzung wird urheberrechtlich verfolgt.

This document is for scientific use only (license CC BY-NC-ND), commercial use of copyrighted material will be prosecuted. 


\title{
AIAKEION
}

\section{Beiträge zur Klassischen Altertumswissenschaft}

\author{
zu Ehren von \\ Florens Felten
}

herausgegeben von

Claus Reinholdt - Peter Scherrer - Wolfgang Wohlmayr

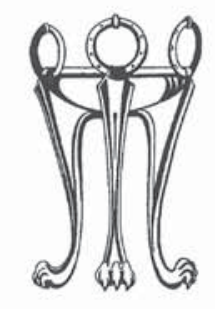

Phoibos Verlag, Wien 2009 
Gedruckt mit Unterstützung durch:

Paris Lodron-Universität Salzburg

Amt der Salzburger Landesregierung

Österreichisches Archäologisches Institut

Bibliografische Information Der Deutschen Bibliothek

Die Deutsche Bibliothek verzeichnet diese Publikation in der Deutschen Nationalbibliografie; detaillierte bibliografische Daten sind im Internet über http://dnb.ddb.de abrufbar.

Bibliographic information published by Die Deutsche Bibliothek

Die Deutsche Bibliothek lists this publication in the Deutsche Nationalbibliografie;

detailed bibliographic data is available in the Internet at http://dnb.ddb.de.

Einband: Apulischer Volutenkrater mit Unterweltsszene (Detail), München Antikensammlung Inv. 3297, Museumsaufnahme HL A 429; Einbandgestaltung: M. Del-Negro, C. Reinholdt

Copyright (C) 2009, Phoibos Verlag, Wien. All rights reserved www.phoibos.at; office@phoibos.at

Printed in Austria: Robitschek \& Co. Ges.m.b.H., 1050 Wien

ISBN 978-3-85161-026-0 


\section{INHALT}

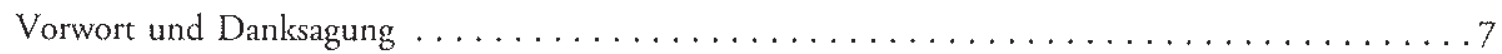

Joachim Dalfen (Salzburg)

„Aus Dir alles, in Dir alles, in Dich alles". Marc Aurel - Die Religion eines römischen Kaisers

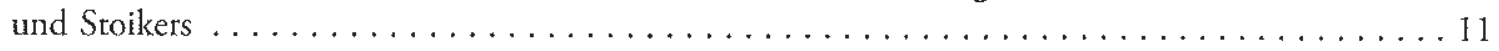

Hans Rupprecht GoETTE (Berlin)

Choregische Weihgeschenke Athens in architektonischer Form (Kurzfassung) . . . . . . . . 19

Herbert Grassl (Salzburg)

Ein Dichter, ein Sklave und ein Hund. Äginetische Gedankenspiele . . . . . . . . . . . 21

Stefan Hiller (Salzburg)

Aiakiden im Diesseits. Der panhellenische Kosmos der Nachkommen des Aiakos . . . . . . . . 24

Hans Peter IsLer (Zürich)

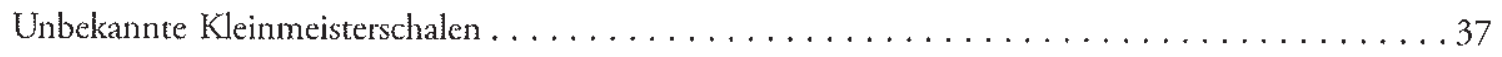

Sigrid JALKoTzY (Salzburg)

Was geschah in Aigina während und nach der mykenischen Palastzeit? . . . . . . . . . . 49

Georgia KoKKorou-Alevras (Athen)

Der antike Demos von Halasarna auf Kos. Vorläufiger Bericht über die Ergebnisse der

Ausgrabungen und des Surveys.................................. 59

Thuri Lorenz (Graz)

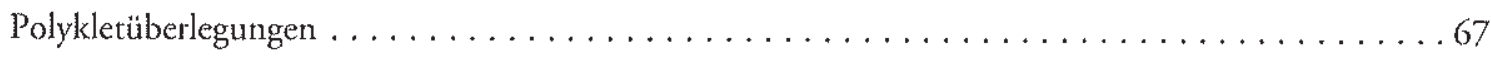

Wolfram Martini (Giessen)

Ein Krieg zwischen Ägina und Athen und seine modischen Folgen............... 75

Marion Meyer (Wien)

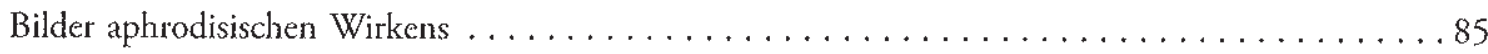

Brinna Otro (Innsbruck)

Frühe Spuren der Aphrodite auf Zypern . . . . . . . . . . . . . . . . . . 99

Eduard Pollhammer (Salzburg)

Andron-Architektur auf Ägina-Kolonna . . . . . . . . . . . . . . . . . . . 113

Thomas Schroren (Salzburg)

„Bewegte Bilder". Zur Entstehung des Kunstwerks bei dessen Beschreibung . . . . . . . . . 129

Gerda Schwarz (Graz)

Hera im trojanischen Krieg. . . . . . . . . . . . . . . . . . . 143

Eva Srmantoni-Bournia (Athen)

The Aeginetan versus a West Saronic Gulf pottery workshop . . . . . . . . . . . . 153

Wolfgang Speyer (Salzburg)

Das 'Märchen' von Amor und Psyche als Offenbarungstext mit einem Ausblick auf Goethes

'Selige Sehnsucht' . . . . . . . . . . . . . . . . . . . . . . . . . . 161

Elisabeth WALDE (Innsbruck)

Eine laurentische Sau in Sperlonga? . . . . . . . . . . . . . . . . . . 171

Tafelteil 


\title{
Was geschah in Aigina während und nach der mykenischen Palastzeit?*
}

\author{
Sigrid Deger-Jalkotzy (Wien)
}

Die im Titel gestellte Frage ist nicht neu. Zu bedeutend war die stolze, mächtig befestigte prähistorische Stadt auf dem Kolonna-Hügel während des Mittelhelladikum und in der frühmykenischen Periode ${ }^{1}$, als dass man sich nicht Gedanken darüber gemacht hätte, weshalb Aigina diese überregionale Geltung in der mykenischen Palastzeit anscheinend nicht halten konnte, und besonders, wie und warum um 1200 v. Chr. (oder kurz danach) die Siedlung ihr Ende fand ${ }^{2}$. Vielfach wurde darauf hingewiesen, dass Siedlungsschichten und Strukturen des 14. und 13. Jhs. v. Chr. mit großer Wahrscheinlichkeit der späteren Bautätigkeit ab der archaischen Periode zum Opfer gefallen seien: Immerhin boten die Funde aus den Gräbern auf dem sog. Windmühlenhügel, die mit größter Wahrscheinlichkeit zur Siedlung von Aigina-Kolonna gehört hatten, hinreichend Nachweise für eine andauernde Besiedlung der Stadt in SH IIIA und IIIB ${ }^{3}$. Bestätigt hat sich diese Sichtweise durch die Ergebnisse der neueren Ausgrabungen am Südhang des Siedlungshügels, wo unter den archaischen und hellenistischen Ruinen die Reste von mykenischen Terrassenmauern und Bauten gefunden wurden wie auch reichlich Keramik aus SH IIIA und SH IIIB ${ }^{4}$. Demnach darf man annehmen, dass sich im Laufe der Palastzeit die Bebauung des Hügels auch nach Süden in Richtung Meer hin ausweitete und Aigina-Kolonna eine Unterstadt besaß, die sich bis zum Hafen ausdehnte ${ }^{5}$. Der Keramikchronologie zufolge erreichte die palastzeitliche Siedlung ihr „Floruit“ in SH IIIA2 und IIIB1 ${ }^{6}$.

* Gewidmet ist dieser Beitrag Florens und Vassiliki Felten, die ich beide vor langer Zeit auf Aigina kennen lernte und mit denen ich mich freundschaftlich verbunden fühle. Folgenden Personen sei herzlich Dank gesagt: F. Felten für klärende Auskünfte zu den Grabungen auf Aigina und für die Genehmigung, unpublizierte Befunde in meine Argumentation aufzunehmen, C. Reinholdt für Hinweise auf neue Befunde und ihre Chronologie, W. Gauß und R. Smetana für Diskussionen über Aigina, M. del Negro für Hilfe mit den Abbildungen; Y. Lolos und A. Vlachopoulos sei für Unterstützung bezüglich der Abbildungen von Kanakia gedankt.

I Vgl. zuletzt F. Felten, Aegina-Kolonna. The History of a Greek Acropolis, in: F. Felten - W. Gauß R. Smetana (Hrsg.), Middle Helladic Pottery and Synchronisms (= Ägina-Kolonna. Forschungen und Ergebnisse, Band I, herausgegeben von F. Felten), Proceedings of the International Workshop held at Salzburg Oct. $31^{\text {st }}-$ Nov. $2^{\text {nd }}, 2004$ (Wien 2007) 11-34. - Zur überregionalen Bedeutung Aiginas insbesondere von MH II an und während der frühmykenischen Periode vgl. die Beiträge von J. B. Rutter, A. Ph. Touchais, M. Lindblom, K. Sarri und J. Maran im selben Kongressband. - Vgl. ferner C. Zerner, New Perspectives on Trade in the Middle and Early Late Helladic Periods on the Mainland, in: C. Zerner et al. (Hrsg.), Wace and Blegen. Pottery as Evidence for Trade in the Aegean Bronze Age 1939-1989. Proceedings of the International Conference held at the American School of Classical Studies at Athens, December 2-3, 1989 (Amsterdam 1993) 39-56. - J. B. Rutter, The Prepalatial Bronze Age of the Southern and Central Greek Main- land, in: T. Cullen (Hrsg.), Aegean Prehistory. A Review (Boston 2001) 95-147 (zu Aigina 125-130). M. Lindblom, Marks and Makers. Appearance, Distribution and Function of Middle and Late Helladic Manufacturers' Marks on Aiginetan Pottery (Jonsered 2001) passim.

2 Vgl. P. Alin, Das Ende der Mykenischen Fundstätten auf dem Griechischen Festland (Lund 1962) 114-115. - St. Hiller, Alt-Ägina IV/1. Mykenische Keramik (Mainz am Rhein 1975) 55-56. - F. Schachermeyr, Die ägäische Frühzeit, Band 2: Die mykenische Zeit und die Gesittung von Thera (Wien 1976) 98.

3 Hiller 1975 (wie Anm. 2) 9. 55-56. - Felten 2007 (wie Anm. 1) 18. - Für palastzeitliche Keramik aus Aigina siehe auch P. A. Mountjoy, Regional Mycenaean Decorated Pottery (Rahden/Westfalen 1999) 493-499.

4 F. Felten - St. Hiller - C. Reinholdt - R. Smetana - W. Gauß, Ägina-Kolonna 2002, ÖJh 72, 2003, 64 f. St. Hiller, Keramik mit Pictorial Style-Dekor aus Ägina Kolonna, in: E. Rystedt - B. Wells (Hrsg.), Pictorial pursuits. Figurative painting on Mycenaean and Geometric pottery. Papers from two seminars at the Swedish Institute at Athens in 1999 and 2001 (Stockholm 2006) 73-82.

5 Felten - Hiller - Reinholdt - Smetana - Gauß, wie Anm. 4.

6 Vgl. auch St. Hiller, Some preliminary thoughts about Aegina in the Dark Ages, in: E. Konsoláki-Giannopoúlou (Hrsg.), Argosaronikós. Praktiká prótou diethnoús synedríou istorías kai archailogías tou Argosaronikoú, Poros, 26-29 Iouniou 1998 (Athen 2003) 11-20. - F. Felten und C. Reinholdt haben mir Einsichtnahme in die Photos der neuen Fundkeramik gewährt. 
Auch die mykenischen Befestigungsmauern im NO-Bereich der Akropolis sollten davor warnen, Aiginas Rolle während der mykenischen Palastzeit zu unterschätzen (Taf. XIII, Abb. 12). Der zugehörigen Fundkeramik nach wurde die Anlage in frühmykenischer Zeit errichtet ${ }^{7}$. Sie hatte aber drei Phasen, deren Chronologie durch Nachuntersuchungen in jüngerer Zeit insgesamt von der Schachtgräberzeit bis in SH IIIB-Mitte gesichert werden konnte ${ }^{8}$.

Die Chronologie der Befestigungsanlage stimmt demnach mit jener der Siedlungsreste vom Südhang des Hügels überein. Aigina-Kolonna war offenbar auch noch während des 14. und 13. Jhs. v. Chr. eine ausgedehnte Stadt, die zumindest zur Landseite hin befestigt war. Zu jener Zeit waren Siedlungen dieser Größenordnung auf den nahe dem Festland gelegenen Inseln und auf den Kykladen selten. Am ehesten vergleichbar ist die mykenische Stadt von Phylakopi auf Melos, die von einem Herrschersitz dominiert wurde. Ob sich auch in Aigina-Kolonna eine vergleichbare Residenz mit entsprechender Architektur befand, muss angesichts des Bau- und Fundverlustes durch die spätere Überbauung offen bleiben. Ausschließen kann man die Existenz eines politischen Zentrums in Kolonna jedoch nicht. In diesem Zusammenhang gewinnt einer der unscheinbarsten Funde der neueren Grabungen im Bereich 'Südhügel' an Bedeutung, den W. Gauß gebührend gewürdigt hat: Es ist dies eine zerbrochene Tonplombe vom Typus der 'irregular noduli', Verwaltungsinstrumente des internen Güterverkehrs mykenischer Palast-Staaten, die bisher ausschließlich in mykenischen Palästen ans Licht gekommen waren. Sollten die geplanten chemischen Analysen beweisen, dass dieses Objekt tatsächlich aus lokalem Ton angefertigt wurde?, dann wurde es auf der Insel nicht nur geöffnet, sondern stammte sogar von dort. Den Fundumständen nach ist es in SH IIIA zu datieren ${ }^{10}$. Wer immer diese Plombe in Aigina öffnete, war dazu berechtigt. Dass es sich dabei um einen Vertreter (Statthalter?, Gouverneur?) eines mykenischen Palast-Staates handelte, Aigina also zum Herrschaftsgebiet eines Palastes gehörte, kann zumindest nicht ausgeschlossen werden ${ }^{11}$. Mit seiner dem Festland nahen Lage im Saronischen Golf musste die Insel für die Paläste der Argolis und wahrscheinlich (über die Kithairon-Route) auch für Theben als wichtige Station auf den Seewegen nach Kreta ebenso wie nach dem Osten interessant gewesen sein. Ich neige aber eher zur Annahme, dass Aigina-Kolonna im 14. Jh. v. Chr. immer noch eine eigenständige Rolle im Netzwerk der überseeischen Handelsbeziehungen spielte, so etwa als Exporteur des weithin begehrten Kochgeschirrs ${ }^{12}$. Vielleicht nicht zufällig wurde die Tonplombe in „unmittelbarer Nähe“ eines in SH IIIA zu datierenden Töpferofens gefunden ${ }^{13}$. Darüber hinaus ergibt sich aus der Ausdehnung der Unterstadt bis zum Hafen, dass Aigina-Kolonna auch im 14. Jh. v. Chr. ein bedeutendes Handelszentrum gewesen sein musste, an dem Güter zusammenliefen und verschifft wurden.

In die gleiche Richtung weisen die mykenischen Siegel, die beim Aphaia-Tempel gefunden wurden, wo sich nach einhelliger Meinung der Forschung auch während der Bronzezeit ein Heiligtum befunden haben dürfte ${ }^{14}$. Die Zahl der Siegel ist ungewöhnlich groß ${ }^{15}$. Zum überwiegen-

7 Vgl. W. Wohlmayr, Ägina-Kolonna. Die schachtgräberzeitliche Siedlung, in: R. Laffineur (Hrsg.), Transition. Le monde égéen du Bronze moyen au Bronze récent (Liège 1989) 151-153. - ders., Schachtgräberzeitliche Keramik aus Ägina, in: F. Blakolmer (Hrsg.), Österreichische Forschungen zur ägäischen Bronzezeit 1998. Akten der Tagung am Institut für Klassische Archäologie der Universität Wien, 2.-3. Mai 1998 (Wien 2000) 127-136. - Vgl. auch W. Gauß, Aigina Kolonna in frühmykenischer Zeit, in: E. Alram-Stern - G. Nightingale (Hrsg.), Keimelion. Elitenbildung und elitärer Konsum von der mykenischen Palastzeit bis zur homerischen Epoche. Akten des internationalen Kongresses vom 3. bis 5. Februar 2005 in Salzburg (Wien 2007) 163-172.

8 Freundliche Auskunft von F. Felten und C. Reinholdt. - Neuester Plan bei W. Wohlmayr, Aegina MH III
- LH I: Ceramic Phase of an Aegean Trade-Domain, in: Felten - Gauß - Smetana 2007 (wie Anm. 1) 45-55, Fig. 1.

9 Dazu Gauß 2007 (wie Anm. 7).

Io Gauß 2007 (wie Anm. 7) 166-167.

I I In SH IIIA1 käme sogar noch der mykenische Palast von Knossos in Frage.

I2 Die Töpfe wurden noch auf der Insel mit eigenen Töpfermarken versehen, vgl. Lindblom 2001 (wie Anm. 1). I3 Gauß 2007 (wie Anm. 7) 166.

I4 I. Pini, Aegina, Aphaia-Tempel. X. Die Steinsiegel. AA 1987, 413-433. - A. Dickers, Die spätmykenischen Siegel aus weichem Stein. Untersuchungen zur spätbronzezeitlichen Glyptik auf dem griechischen Festland und in der Ägäis (Rahden/Westfalen 2001) 149-156.

I5 Vgl. O. Krzyszkowska, Aegean Seals. An Introduction (London 2005) 275. 
den Teil handelt es sich um Weichstein-Siegel der so genannten „Mainland Popular Group“16. Allgemein wird dieser Typus der Palastzeit von SH IIIA und IIIB zugeschrieben. Nach A. Dickers, die den mykenischen Weichsteinsiegeln eine Monographie gewidmet hat, gehörten die Träger solcher Siegel in Palast-Staaten eher den unteren Rängen der Beamtenhierarchie an. Ganz im Gegensatz dazu scheint in jenen Regionen, die nicht von Palästen beherrscht wurden, der Besitz von Siegeln der „Mainland Popular Group“ sozialen Oberschichten vorbehalten gewesen zu sein, die sich an den „... Repräsentationsformen an den Zentren der mykenischen Welt orientierten“, wie Dickers formuliert ${ }^{17}$. Nach Ansicht von B. Eder waren die Weichsteinsiegel der „Mainland Popular Group“ ebenso wie Siegel aus gepresstem Glas und aus Fluorit ihrer Funktion nach eher Instrumente der mykenischen Paläste. Sie seien zum Zweck der administrativen Erschließung auch jener Regionen eingesetzt worden, die außerhalb der palaststaatlichen Territorien lagen ${ }^{18}$. Tatsächlich scheinen sich in der Verbreitung von Bildthemen und von stilistisch miteinander verwandten Gruppen der Siegel gewisse Muster interregionaler Beziehungen zu spiegeln ${ }^{19}$. Es könnte sich sehr gut um Netzwerke wirtschaftlicher und wohl auch politischer Beziehungen gehandelt haben, die sich in den Regionen außerhalb der Palast-Staaten etablierten und die von den Angehörigen lokaler Eliten getragen wurden. Es ist gut vorstellbar, dass sich die Paläste solcher lokaler Netzwerke für ihre wirtschaftlichen Interessen bedienten, so etwa bei der Beschaffung landwirtschaftlicher Produkte (Getreide!), oder zur Sicherung von Geleitschutz für Personen und Güter bei Transporten zu Land und zur See. In dieser Sicht können Siegel nicht nur für Kooperationen zwischen „peripheren“ Regionen außerhalb der Palast-Staaten zeugen, sondern auch für Kooperationen zwischen Palästen und lokalen Herrschaftsträgern. Ansichtssache bleibt es hingegen, ob man aus den Siegeln als einzigem Indiz die These ableiten darf, dass die Paläste auch jene Territorien, die außerhalb ihrer Staatsgrenzen lagen, unter wirtschaftlicher Kontrolle hielten ${ }^{20}$.

Stellen wir also die Siegel vom Typus der „Mainland Popular Group“ von Aigina in diesen Rahmen. So gut wie alle Stücke sind abgewetzt, was bedeutet, dass sie einstmals tatsächlich gebraucht worden waren. Sie waren daher nicht eigens angefertigte Opfergaben, sondern sie wurden von ihren Besitzern in Heiligtümern deponiert, als sie ihrer nicht mehr bedurften ${ }^{21}$. Die Weichsteinsiegel von Aigina tragen Bilder, die sich, wenngleich in stilistisch sehr unterschiedlichen Ausprägungen, auf Siegeln aus praktisch allen mykenischen Regionen des Festlandes bis nach Thessalien wieder finden ${ }^{22}$. Stilistisch enger verwandt sind einige Siegelbilder von Aigina mit solchen aus der Korinthia und aus der Argolis ${ }^{23}$. Daraus folgt, dass Aigina während der mykenischen Palastzeit des 14. und 13. Jhs. v. Chr. einerseits in ein regional weit gespanntes Netzwerk von Beziehungen integriert war, gleichzeitig aber in enger Kooperation mit den Palästen der Argolis stand. Die Situation am westlichen Kap der Insel weist ja geradezu in die Richtung von Epidauros und Isthmia bzw. Korinthia, und damit zu wichtigen Stationen eines Straßensystems,

I6 Pini 1987, Dickers 2001 (wie Anm. 14): Drei Hartsteinsiegeln der Perioden SH I - IIIA stehen 33 Weichsteinsiegel aus SH IIIA2 - IIIB gegenüber. Von diesen stammen 31 vom Aphaia-Tempel, 2 weitere allgemein aus Aigina.

I7 Dickers 2001 (wie Anm. 14) 115-117.

I8 B. Eder, Im Spiegel der Siegel. Die nördlichen und westlichen Regionen Griechenlands im Spannungsfeld der mykenischen Paläste, in: E. Alram-Stern - G. Nightingale (Hrsg.) 2007 (wie Anm. 7) 81-124.

I9 Siehe die Verbreitungskarten bei Dickers 2001 (wie Anm. 14) Karten 3-10; Eder 2007 (wie Anm. 18) Abb. 6-8.

20 Entschieden zu weit geht es m. E., wenn aus der Verteilung der Weichstein-, Glas- und Fluorit-Siegel die Ausweitung einer staatlichen Souveränität der Paläste über den gesamten festländischen Bereich der mykenischen Kultur abgeleitet wird. Damit würde man mit nach wie vor ungenügenden Argumenten wieder bei der alten Vorstellung von einem 'Mycenaean Empire' landen.

2 I Gleiches gilt für die Weichsteinsiegel von anderen spätbronzezeitlichen Heiligtümern, vgl. O. Krzyszkowska 2005 (wie Anm. 15) 275. 209. - Für die gut datierte Fundgruppe aus Phylakopi siehe J. G. Younger, The sealstones, in: C. Renfrew, The Archaeology of Cult. The Sanctuary at Phylakopi (London 1985) 281-297.

22 Dickers 2001 (wie Anm. 14) Karte 2 (Vierfüßer), Karten 3, 7 (Ornamente).

23 Dickers 2001 (wie Anm. 14) Karte 6 (Tierdarstellungen der 'Gruppe 1'). 
in dessen Zentrum die Paläste der Argolis lagen ${ }^{24}$. Getragen wurden die überregionalen Beziehungen Aiginas von einer sozialen Elite, die für uns durch die Inhaber der Weichsteinsiegel fassbar wird und die man sich kaum anderswo angesiedelt vorstellen kann als in der Stadt von Kolonna. Gemessen an der Tatsache, dass beim Aphaia-Tempel eine der größten Ansammlungen mykenischer Siegel der „Mainland Popular Group“ gefunden wurde ${ }^{25}$, könnte es sich sogar um eine relativ große Personengruppe gehandelt haben.

Vom späteren 14. Jh. v. Chr. an und während des Großteils des 13. Jhs. v. Chr. (SH IIIA2 und IIIB1) scheint sich Aigina kulturell zunehmend in die von den Palastzentren dominierte, so genannte mykenische Koiné eingegliedert zu haben. Darauf deuten jedenfalls stilistische und naturwissenschaftliche Analysen der Feinkeramik hin ${ }^{26}$. Doch ähnlich wie im Fall der Kykladen ${ }^{27}$ muss mit dem zunehmenden kulturellen Einfluss durch die Palastkultur nicht zugleich auch die politische Dominanz der Paläste über Aigina einhergegangen sein. Eher ist zu vermuten, dass sich die ökonomischen Aktivitäten der Bewohner von Aigina in einer Zeit, in der das Palastsystem seinen politischen und ökonomischen Höhepunkt erreichte, stärker als zuvor an den Interessen und am Bedarf der Paläste orientierten.

Im Gegensatz zu SH IIIA und den frühen Phasen von SH IIIB ist die ausgehende Palastzeit (SH IIIB2) in Aigina-Kolonna spärlich belegt ${ }^{28}$, selbst wenn man die Absenz der typischen Keramikformen dieser Phase damit erklären könnte, dass die keramischen Trends der palastzeitlichen Keramik in den Regionen außerhalb der Palast-Staaten nicht mehr mitgemacht wurden. Die Stadt von Aigina-Kolonna scheint gegen Ende der Palastzeit tatsächlich einen Niedergang erlebt zu haben.

Genau um diese Zeit entstand an der SW-Küste der Nachbarinsel Salamis bei Kanakia eine große urbane Anlage mit dichter Bebauung und einem Netzwerk von Verkehrswegen, die vor wenigen Jahren von Y. G. Lolos entdeckt und ausgegraben wurde ${ }^{29}$. In ihrem Umkreis wurde außerdem eine Infrastruktur aus kleineren dörflichen Ansiedlungen nachgewiesen ${ }^{30}$. Die Lage einer mykenischen Stadt im Westen von Salamis ist umso bemerkenswerter, als sich nachmykenische Siedlungen und Nekropolen der Insel eher auf der östlichen Seite, also Athen zugewandt, fin$\operatorname{den}^{31}$. Die Orientierung nach Westen ist etwas, das Kanakia mit der Siedlung von Aigina-Kolonna teilte. Dass auch im Fall von Kanakia diese geographische Ausrichtung mit maritimen Aktivitäten, und insbesondere mit den Handelsbeziehungen der Paläste, zusammenhing, bezeugen zwei Hafenbuchten ebenso wie einige importierte Objekte östlicher Herkunft im Fundmaterial (dazu weiter unten).

Wurde der Niedergang von Aigina-Kolonna gegen Ende des 13. Jhs. v. Chr. durch den Aufstieg einer Rivalin in Salamis ausgelöst, die noch dazu in Sichtweite lag? Die Diskussion dieser Frage setzt zunächst eine zumindest generelle Beschreibung von Kanakia voraus. Von der Unterstadt an den beiden erwähnten Häfen führte eine gepflasterte, axial durch die Siedlung verlaufende Straße zum Zentrum auf den beiden miteinander verbundenen, als „Akropolis“ bezeichneten Anhöhen ${ }^{32}$, wo mehrere architektonisch aufwendige Großstrukturen lagen.

24 Vgl. R. Hope Simpson - D. K. Hagel, Mycenaean Fortifications, Highways, Dams and Canals (Sävedalen 2006) $148-156$.

25 Vgl. Krzyszkowka 2005 (wie Anm. 15) 275.

26 Hiller 1975 (wie Anm. 2) 54-55. - Gauß 2007 (wie Anm. 7) 166.

27 Vgl. A.-L. Schallin, Islands under Influence. The Cyclades in the Late Bronze Age and the Nature of Mycenaean Presence (Jonsered 1993) zusammenfassend 188189.

28 Hiller 2006 (wie Anm. 4). - P. A. Mountjoy 1999 (wie Anm. 3) 495.

29 Die folgenden Ausführungen orientieren sich an dem Bericht von Y. G. Lolos, Mikinaikí Sálamis: I érevnes tón etón 2000-2002, Dodoni. Istoría kai archailogía 32, 2003, 17-179. - Für eine kurze Zusammenfassung siehe auch Y. G. Lolos, Salamis, in: A. G. Vlachopoulos (Hrsg.), Archaeology. Aegean Islands (Athen 2006) 176-181 (über Kanakia 178-180).

30 Lolos 2003 (wie Anm. 29) 20.

3 I Dazu vgl. Y. G. Lolos, Dark Age Citadels in Southern Salamis, in: V. Karageorghis - Chr. E. Morris (Hrsg.), Defensive Settlements of the Aegean and the Eastern Mediterranean after c. 1200 B.C. (Nicosia 2001) 115-136.

32 Lolos 2003 (wie Anm. 29) 20. 38 Abb. 15. 16. 
Bau Gamma war ein großer, in zwei Flügeln angelegter Wohnkomplex ${ }^{33}$. Wichtigstes Element war ein lang gestreckter, mehrfach unterteilter Raum großen Ausmaßes, dessen Vorraum im Eingangsbereich eine steinerne Säulenbasis aufwies (Taf. XIII, Abb. 3). Obschon vom Ausgräber als „Megaron“ bezeichnet, entspricht die Raumgruppe dem Typus des kanonischen Megarons mykenischer Palastanlagen jedoch nicht ${ }^{34}$. Den Charakter einer herrschaftlichen Residenz wird man ihr freilich ohne weiteres zusprechen. Vielleicht eignet sich anstelle von „Megaron“ der griechische Begriff „Melathron“ besser zur Bezeichnung derartiger Anlagen ${ }^{35}$. Unter den Funden aus Bau Gamma fällt ein großer, mit Ritzdekor ausgestatteter Konulus aus Steatit auf ${ }^{36}$, der eher kein einfacher „Spinnwirtel“ war, sondern ein Rangabzeichen oder ein Statussymbol ${ }^{37}$. Ein ganz ähnliches Objekt wurde kürzlich auch auf Aigina gefunden, und zwar in einem mykenischen Kammergrab bei Lazarides im Ostteil der Insel ${ }^{38}$.

Ein weiteres Gebäude größeren Ausmaßes, möglicherweise ein Apsidenhaus, erbrachte Hinweise auf kultische Aktivitäten ${ }^{39}$. Wirtschaftlichen Zwecken diente es anscheinend nicht.

Größtes Interesse verdient jedoch ein ausgedehnter Komplex, bestehend aus drei Bauten mit insgesamt über 40 Räumen ${ }^{40}$. Sie zogen sich entlang von Korridoren auf zwei bis drei unterschiedlichen Niveaus des terrassierten Hanges hin (Taf. XIII, Abb. 4), entsprachen demnach dem Typus der palastzeitlichen „mykenischen Korridorhäuser “41. Die Anlage beherbergte ausschließlich Werkstätten und Lagerräumlichkeiten mitsamt aller möglichen Nebenräume, weshalb Y. Lolos von einem ,industrial complex“ spricht. Der Hauptzugang, ein doppeltes, mit einem Turm zusätzlich gesichertes Tor, lag im Westen (Taf. XIII, Abb. 5). In diesem Bereich fanden sich zudem Hinweise auf den Gebrauch von Pfeilen und Steinschleudern. Zusammen mit den massiven Steinmauern des gesamten Komplexes deutet alles darauf hin, dass dem Schutz dieser Anlage groBes Augenmerk gewidmet wurde.

$\mathrm{Zu}$ den Funden aus diesem Wirtschaftskomplex gehören Fragmente von mindestens fünf im Allgemeinen als Badewannen (asaminthoi) interpretierten Großbehältern aus Ton, also von Gegenständen des gehobenen Wohnstandards der Palastkultur. Dass sie in Kanakia nicht im Wohnbereich, sondern im Wirtschaftskomplex gefunden wurden, ist im mykenischen Kulturraum ohne Parallele ${ }^{42}$. Handelte es sich um Werkstücke, die an andere Lokalitäten innerhalb der Siedlung transportiert werden sollten? Allerdings lässt der Fund eines Weichsteinsiegels ${ }^{43}$ darauf schließen, dass sich auch im Wirtschaftstrakt Personen höheren Ranges aufhielten. Oder waren die Wannen gar für den Export bestimmt? Der Ausgräber hat diese Frage bisher offen gelassen.

33 Lolos 2003 (wie Anm. 29) 76-79; engl. Zusammenfassung 81-82.

$34 \mathrm{Zu}$ diesem Thema vgl. R. Jung, Das Megaron - ein Analogie(kurz)schluss der ägäischen Archäologie, in: A. Gramsch (Hrsg.), Vergleichen als archäologische Methode: Analogien in den Archäologien (Oxford 2000) 71-95.

$35 \mathrm{Zu}$ diesem Begriff vgl. Sp. I. Iakovidis, Die Festung zu Gla, Wohnsitz von zwei Mitgliedern der Orchomenischen Elite, in: E. Alram-Stern - G. Nightingale (Hrsg.) 2007 (wie Anm. 7) 209-217.

36 Lolos 2003 (wie Anm. 29) 79. 95 Abb. 22. - Lolos 2006 (wie Anm. 29) 179 und Abb. 238.

37 Für eine zusammenfassende Darstellung mit Literaturhinweisen vgl. E. Alram-Stern, Konuli, in: E. AlramStern - S. Deger-Jalkotzy (Hrsg.), Aigeira I. Die mykenische Akropolis, Faszikel 3 (Wien 2006) 130-133. - Ritzverzierte Konuli aus Steatit werden eher der weiblichen als der männlichen Repräsentations-Sphäre zugeschrieben. Der Fund des Stückes von Kanakia in einem Wohnbereich muss kein Widerspruch sein.
38 Abgebildet auf einem Plakat, das N. Sgouritsa 2006 bei der Tagung Mesohelladika in Athen vorstellte, vgl. www. Mesohelladika.com: Lazarides on Aegina: Another Prehistoric Site.

39 Vgl. Lolos 2003 (wie Anm. 29) 77. 82. 94 Abb. 20 (Terrakotta-Figurinen vom Phi- und Psi-Typ und Tierfigürchen).

40 Lolos 2003 (wie Anm. 29) 48-55. 76-79; engl. Zusammenfassungen 20-22. 81-82.

4 I Lolos 2003 (wie Anm. 29) 50, mit weiteren palastzeitlichen Beispielen. Allgemein zum mykenischen 'Korridorhaus' und seinen Sonderformen vgl. G. Hiesel, Späthelladische Hausarchitektur (Mainz am Rhein 1990) 111-158.

42 Lolos 2003 (wie Anm. 29) 136-139. 178-179 Abb. 37-39. Lolos verweist auf palastzeitliche Badewannen aus Messenien, Argolis, Attika, Theben und Dimini, sowie auf Exemplare aus SH IIIC von Paros und Naxos.

43 Lolos 2003 (wie Anm. 29) 94 Abb. 19. - Zur Bedeutung der mykenischen Weichsteinsiegel vgl. oben, S. 51, zum Siegel aus Kanakia weiter unten, S. 56. 
Die beiden Hafenbuchten und die breite Straße zur Akropolis bezeugen, welche Bedeutung die Verbindungen zur See für das Wirtschaftszentrum von Kanakia hatten. Zwar waren die beweglichen Funde aus den Gebäuden der Akropolis eher bescheiden. Wahrscheinlich nahmen die Bewohner beim Verlassen der Siedlung ihre Wertgegenstände mit (zum Ende des mykenischen Kanakia siehe weiter unten). Trotzdem blieben einige Gegenstände zurück, die Kanakia als wichtige Station im spätbronzezeitlichen Güterverkehr zur See erscheinen lassen ${ }^{44}$. Neben Keramikerzeugnissen aus der Argolis und aus Rhodos fanden sich Stücke aus grauem Andesit und Kochtöpfe mit eingeritzten Töpferzeichen, beides aus Aigina. Bemerkenswert sind weiters Zeugnisse für Verbindungen mit Zypern, so das Fragment eines zyprischen Kupferbarrens und möglicherweise ein Fragment einer zyprischen Wandapplik ${ }^{45}$.

Anders als der Ausgräber würde ich das Ensemble der Bauten auf der „Akropolis“ von Kanakia nicht als Palast bezeichnen, weil charakteristische Elemente wie kanonisches Megaron, aufwendige Ausstattung der Innenräume und vor allem Zeugnisse für den Gebrauch von Schrift fehlen. Weiters kann das agrarische Einzugsgebiet der Insel keine ausreichende Grundlage für den Unterhalt einer mykenischen Palastorganisation mit ihren komplexen sozialen, politischen und wirtschaftlichen Strukturen geboten haben. Jedoch wird man dem Ausgräber zustimmen, dass Kanakia seinen eindrucksvollen Anlagen zufolge als ein politisches und wirtschaftliches Zentrum gesehen werden muss, das von einem starken lokalen Herrschaftsträger, vielleicht sogar von einer lokalen Dynastie, kontrolliert wurde.

Was lässt sich also aus den Befunden von Kanakia im Hinblick auf die palastzeitliche Entwicklung von Aigina-Kolonna ableiten? Zunächst meine ich, dass die mächtige Siedlung von Kanakia eine Vorstellung davon vermittelt, wie Aigina-Kolonna im 14. und 13. Jh. v. Chr. ausgesehen haben mag. Neben den Befestigungsmauern, von denen immerhin noch Reste erhalten sind, darf man sich ausgedehnte Wirtschaftsgebäude ebenso vorstellen wie große Wohnanlagen gehobenen Standards. Architektonisch könnten die repräsentativen Bauten ebenfalls dem palastzeitlichen „Korridorhaus“-Typ entsprochen haben, der beispielsweise in Mykene vom, House of The Oil Merchant" und den drei benachbarten Häusern auf den Terrassen außerhalb der Burgmauer $^{46}$ oder in Tiryns von den Bauten in der Unterburg ${ }^{47}$ repräsentiert wurde. Es ist $\mathrm{m}$. E. wahrscheinlich, dass Aigina-Kolonna in SH IIIA-IIIB1 tatsächlich ein politisches Zentrum mit einem „Melathron“ war, so wie Phylakopi und wie etwas später Kanakia.

Ein wichtiger Unterschied zwischen Aigina und Kanakia liegt jedoch in der Chronologie der beiden Städte. Zwar wird mykenische Besiedlung von Salamis durch Funde aus mykenischen Kammergräbern der Perioden SH IIIA bis IIIC bezeugt, und auch Kanakia scheint der Fundkeramik zufolge bereits in SH IIIA1 besiedelt gewesen zu sein ${ }^{48}$. Doch wurde den Berichten von Y. Lolos zufolge die mächtige Großanlage erst während der Spätphase der Palastzeit gebaut, um das Ende von SH IIIB1 oder überhaupt erst in SH IIIB2 ${ }^{49}$. Das würde bedeuten, dass die Blütezeit von Kanakia mit dem Niedergang von Aigina zusammenfiel.

Diese Epoche des späten 13. Jhs. v. Chr. (SH IIIB2) war eine Krisenzeit. Das erklärt wohl die aufwendigen Sicherheitsmaßnahmen für den Wirtschaftskomplex von Kanakia, die zusätzlich zu Befestigungsanlagen der Siedlung ${ }^{50}$ gebaut wurden. Kanakia folgte darin wohl dem Beispiel

44 Vgl. Lolos 2003 (wie Anm. 29) 78-79 (Kleinfunde, Zusammenfassung). 21 (Keramik-Importe). 99-139 (Analyse der Fundkeramik).

45 Kupferbarren: Lolos 2003 (wie Anm. 29) 21. 40 Abb. 18. Der Ausgräber weist darauf hin, dass etwa zur selben Zeit das beim Kap Gelidonya untergegangene Schiff eine Ladung von Kupferbarren des gleichen Typus an Bord hatte. - Mögliche Wandapplik: Lolos 2003 (wie Anm. 29) 95 Abb. 21.

46 Zusammenfassend G. E. Mylonas, Mycenae, rich in Gold (Athen 1983) 158-161 mit schematisiertem Plan
Abb. 122.

47 Übersichtsplan bei K. Kilian, Ausgrabungen in Tiryns 1980. Bericht zu den Grabungen, AA 1982, Abb. 23.

48 Lolos 2003 (wie Anm. 29) 99-179. Baureste aus dieser Zeit sind jedoch bisher nicht vorgelegt worden.

49 Nach derzeitigem Forschungsstand gibt es auf Salamis keine Siedlung(en), die als Vorläufer der Stadt von Kanakia gelten könnte(n).

50 Lolos 2006 (wie Anm. 29) 178, mit Hinweis auf eine am Südhang aufgefundene Wehrmauer, sowie Türme, Wächterhäuser und Aussichtsposten. 
der mykenischen Paläste, die zur selben Zeit nicht nur ihre Befestigungen aufwendig verstärkten, sondern insbesondere auch für den Schutz ihrer Wirtschaftsanlagen sorgten ${ }^{51}$. Es ist ein Irrtum, anzunehmen, dass die Erweiterungen der mykenischen Zitadellen gegen Ende der Palastära dem Schutz der allgemeinen Bevölkerung dienten. Sie waren vielmehr für die Sicherheit des Palastsystems, seiner Herrscher und seiner wirtschaftlichen Güter bestimmt ${ }^{52}$. Mit diesen Maßnahmen reagierten die Paläste auf die ersten Anzeichen einer Krise, die schließlich tatsächlich zum Zusammenbruch der gesamten mykenischen Palastkultur führen sollte ${ }^{53}$.

Diese Krise wurde offenbar auch auf den Inseln wahrgenommen. So wurde in SH IIIB2 die Siedlung von Grotta auf Naxos verlassen ${ }^{54}$, in Ayios Andreas auf Siphnos wurde eine befestigte Höhensiedlung angelegt ${ }^{55}$, und auf Phylakopi wurden die Befestigungsanlagen verstärkt ${ }^{56}$. In Rhodos kam es - wohl durch Abwanderung - zu einem Bevölkerungsrückgang, und auch auf Kreta war das spätere 13. Jh. v. Chr. eine Periode von Störungen und Niedergang ${ }^{57}$.

In den Regionen um den Saronischen Golf machte sich die Krise ebenfalls bemerkbar. In Athen wurde die Akropolis ummauert und mit einer aufwendigen Anlage zur Wasserversorgung versehen $^{58}$, im Westen erbauten die Bewohner der Korinthia eine große Mauer quer über den Isthmos von Korinth ${ }^{59}$. Was Wunder also, wenn sich auch die Inselbewohner des Saronischen Golfs vorsahen?

Diese Erwägungen führen mich am Ende dieses Beitrages zur Hypothese, dass die Blütezeit von Kanakia und der Niedergang von Aigina-Kolonna nicht nur synchron waren, sondern ursächlich zusammenhingen. Ich gehe sogar weiter und stelle zur Diskussion, dass es Auswanderer aus Aigina waren, die in den letzten Jahrzehnten des 13. Jhs. v. Chr. die mächtige urbane Anlage in Kanakia erbauten. Diese Ansicht geht zunächst davon aus, dass die Errichtung eines Großzentrums wie Kanakia nur durch eine starke politische Macht mit straffen Führungsstrukturen bewirkt werden konnte. Weiters mussten die Machthaber von Kanakia über ausgezeichnete Außenverbindungen und viel Erfahrung im Übersee-Verkehr verfügt haben. In Aigina waren diese Voraussetzungen seit Jahrhunderten vorhanden, nicht aber - jedenfalls nach dem gegenwärtigen Forschungsstand - in Salamis ${ }^{60}$. Ferner erforderte der für die Bautätigkeiten in Kanakia geleistete Arbeitsaufwand nicht nur eine entsprechend große Menge an Arbeitskräften, sondern auch das technische Können zur Errichtung von Architekturformen, die durchaus mit jenen der Paläste Schritt halten konnten. Auch hier meine ich, dass es plausibler ist, mit Zuwanderungen aus Aigina als mit einer Konzentration einheimischer Bevölkerungsgruppen aus Salamis im Südwesten der Insel zu rechnen. Erstens wurde Monumentalarchitektur in Aigina schon seit dem

5 I Auch die Paläste Pylos und Dimini schotteten im ausgehenden 13. Jh. v. Chr. (SH IIIB2) ihre Magazine und Werkstätten nach außen hin ab. Für Pylos vgl. J. C. Wright, Changes in Form and Function of the Palace at Pylos, in: C. W. Shelmerdine - Th. G. Palaima (Hrsg.), Pylos Comes Alive. Industry + Administration in a Mycenaean Palace (New York 1984) 19-29. - Für Dimini vgl. V. Adrimi-Sismani, The Palace of Iolkos and Its End, in: S. Deger-Jalkotzy - I. S. Lemos (Hrsg.), Ancient Greece From the Mycenaean Palaces to the Age of Homer (Edinburgh 2006) 465-481, mit Übersichtsplan Fig. 25.1.

52 Ausgenommen war vielleicht die Großanlage von Gla in Böotien, wo der riesige östliche Teil des ummauerten Areals ohne Verbauung war. Er könnte tatsächlich als Fluchtburg für den Fall eines Dammbruches und anschließender Überflutung der Kopais gedacht gewesen sein.

53 Eine ausführliche Darstellung bietet mein Abschnitt 15 „Decline, Destruction, Aftermath" in: C. W. Shelmerdine (Hrsg.), The Cambridge Companion to the Bronze Age (New York - Cambridge); im Druck. 54 A. Vlachopoulos, The Late Helladic III C 'Grotta Phase' of Naxos. Its Synchronisms in the Aegean and its Non-Synchronisms in the Cyclades, in: S. Deger-Jalkotzy - M. Zavadil (Hrsg.), LH III C Chronology and Synchronisms. Proceedings of the international workshop held at the Austrian Academy of Sciences at Vienna, May $7^{\text {th }}$ and $8^{\text {th }}$, 2001 (Wien 2003) 217-234.

55 Ch. A. Televantou, Ayios Andreas on Sifnos: a Late Cycladic III Fortified Acropolis, in: V. Karageorghis - Ch.

E. Morris (Hrsg.) 2001 (wie Anm. 31) 191-212.

56 Renfrew 1985 (wie Anm. 21) 81.

57 Deger-Jalkotzy wie Anm. 53.

58 Sp. Iakovidis, Late Helladic Citadels on Mainland Greece (Leiden 1983) 79-82.

59 Aktuell diskutiert bei C. Morgan, Isthmia VIII. The Late Bronze Age Settlement and Early Iron Age Sanctuary (Princeton 1999) 362-365. 437-447. 60 Vgl. Lolos 2003 (wie Anm. 29) 19. 
Mittelhelladikum errichtet, und zweitens dürfte angesichts der Ausdehnung der Stadt von AiginaKolonna auch während der Palastzeit die Bevölkerungszahl entsprechend hoch gewesen sein.

Schließlich sei das früher erwähnte Siegel aus dem Wirtschaftskomplex von Kanakia ins Treffen geführt. Es zeigt einen gehörnten Vierfüßer im Profil sowie vegetabile Füllmotive vor und über dem Tier $^{61}$. Dieses Bildthema ist weit verbreitet, wobei das Horntier in unterschiedlichen Körperhaltungen dargestellt ist ${ }^{62}$. Die größte Gruppe stammt vom Aphaia-Heiligtum in Aigina, wo von den 33 Siegeln der „Mainland Popular Group“ nicht weniger als 22 gehörnte Vierfüßer zeigen ${ }^{63}$. Bemerkenswert ist jedoch, dass davon die Hälfte, d. h. elf Stücke, das Tier in gleicher Haltung wie auf dem Siegel von Kanakia zeigen, nämlich im rechten Profil mit erhobenem Hals und nach vorne gerichtetem Kopf ${ }^{64}$. Die nächst größeren Gruppen von Siegeln mit demselben Motiv stammen aus Mykene (sechs sichere, zwei mögliche Stücke) ${ }^{65}$ und aus Kephallonia (fünf Stücke) ${ }^{66}$. Weitere, zahlenmäßig aber geringere Belege fanden sich in Athen, Euboea, Achaia, in der Korinthia und in Mittelgriechenland. So lässt es auch der Blick auf die Weichsteinsiegel vom Aphaia-Tempel nicht als unmöglich erscheinen, dass in Kanakia ein überregionales Beziehungsnetz genutzt wurde, das zuvor von Aigina aufgebaut worden war und in dem die Paläste der Argolis die Hauptrolle spielten. Der stilistische Vergleich zwischen dem Siegelbild von Kanakia und den übrigen Präsentationen des Motivs muss den Spezialisten für Siegelglyptik überlassen bleiben. Oberflächlich gesehen lässt sich lediglich ein Siegel von Aigina näher zum Vergleich heranziehen ${ }^{67}$, und zwei Siegel von Kokolata auf Kephallonia zeigen die Tierhörner in derselben eigenartigen Weise nach oben gebogen wie auf dem Siegel von Kanakia ${ }^{68}$.

Der Grund, warum Salamis in der Endphase der mykenischen Palastzeit an die Stelle von Aigina als führendes Zentrum im Saronischen Golf getreten sein sollte, lässt sich unschwer erraten: Ägyptischen und vorderasiatischen Schriftquellen zufolge trieben in den letzten Jahrzehnten des 13. Jhs. v. Chr. Piraten im östlichen Mittelmeer ihr Unwesen. Das mag zumindest eine der Ursachen für die früher erwähnten Veränderungen in der Siedlungsstruktur der ägäischen Inseln gewesen sein. Zweifellos sehr exponiert war auch das Kap von Aigina-Kolonna. Die Lage von Kanakia im Südwesten von Salamis war dagegen einerseits geschützter, andererseits aber ebenso geeignet für überseeische Aktivitäten wie das Kap Kolonna.

Zusammenfassend schlage ich daher vor, die Geschichte der mykenischen Stadt von AiginaKolonna während des 13. Jhs. v. Chr. im Kontext mit Kanakia auf Salamis zu sehen. Zu Beginn der Palastzeit in SH IIIA war Aigina wohl immer noch ein bedeutendes autonomes Zentrum im Netzwerk des maritimen Güterverkehrs. In dem Maß, in dem die Paläste während der Zeit von SH IIIA2 und IIIB1 die politische und wirtschaftliche Führungsrolle im mykenischen Griechenland übernahmen, dürfte Aigina, so wie alle anderen nicht-palatialen Regionen auch, zunehmend unter den kulturellen und wirtschaftlichen Einfluss der Palastzentren geraten sein. Das bedeutet jedoch nicht, dass die politische Eigenständigkeit der Insel verloren ging. Dagegen sprechen allein schon die geographischen Gegebenheiten. Auch den archäologischen Befunden nach blieb Aigina-Kolonna die wichtigste Siedlung im Saronischen Golf.

Im späteren 13. Jh. v. Chr. kam es zu einer allgemeinen Krise, die sich vor allem in ersten Zerstörungen mykenischer Palastzentren und anderer Siedlungen äußerte. Danach waren die Paläste, wie erwähnt, vor allem mit der Sicherung ihrer eigenen Anlagen beschäftigt. Es gibt auch

6I Vgl. oben Anm. 43.

62 Dickers 2001 (wie Anm. 14) 20 und Abb. 5; Karte 2.

63 Dickers 2001 (wie Anm. 14).

64 Dickers 2001 (wie Anm. 14) Kat. Nr. 3-5, 9-11, 16,17 ?, 19?, 32, 33 .

65 Dickers 2001 (wie Anm. 14) Kat. Nr. 76-77, 80, 89, 100, 104, 105?, 106?

66 Dickers 2001 (wie Anm. 14) Kat. Nr. 168, 175, $176,185,186$.
67 Dickers 2001 (wie Anm. 14) Kat. Nr. 3.

68 Dickers 2001 (wie Anm. 14) Kat. Nr. 168, 175. Lolos 2003 (wie Anm. 43) bezeichnet das Tier auf dem Siegel von Kanakia als Antilope oder Hirsch. I. Pini, CMS II, 3, S. XXXVII f. weist auf die generelle Problematik der Identifizierung von gehörnten Vierfüßern hin. Dennoch fällt seinen Kriterien zufolge eine Deutung als Hirsch für das Tier auf dem Siegel von Kanakia aus. 
Hinweise, dass der Seehandel nicht mehr ungestört abgewickelt werden konnte. In dieser Situation ergriffen Angehörige der Eliten von Aigina, wie ich glaube, die Initiative und errichteten auf der gegenüberliegenden Küste von Salamis in Kanakia ein neues Machtzentrum, dessen Lage weniger exponiert war als das Kap Kolonna. Tatsächlich deuten die Funde aus Kanakia darauf hin, dass der internationale Güteraustausch noch einige Zeit aufrechterhalten werden konnte.

Die spärlichen Keramikfunde aus SH IIIB2 von Aigina-Kolonna und Funde des späteren SH IIIB von anderen Fundorten der Insel (vor allem Nekropolen) bezeugen, dass Aigina damals nicht vollständig verlassen wurde. Wohl aber dürfte die Bevölkerung dezimiert gewesen sein. Weiters wiesen St. Hiller und P. A. Mountjoy unter den neuen Keramikfunden von Kolonna auch etwa 20 Fragmente aus SH IIIC-Früh ${ }^{69}$ und vielleicht sogar aus SH IIIC-Mitte nach ${ }^{70}$. Dieses Material ist zahlenmäßig nicht viel. Trotzdem ist es wichtig. Bedeutet es doch, dass Aigina-Kolonna entgegen der communis opinio nicht um 1200 v. Chr., sondern erst einige Jahrzehnte später verlassen wurde.

Auch in Kanakia ging nach dem Untergang der mykenischen Paläste um 1200 v. Chr. das Leben während der Periode SH IIIC Früh zunächst weiter. Dann aber wurde die Stadt - anscheinend ohne dass eine Zerstörung stattgefunden hatte - verlassen. Die Bewohner nahmen offenbar ihre Wertgegenstände mit. Nur ein kleiner Hortfund von Bronzen ${ }^{71}$ deutet darauf hin, dass sich sein Eigentümer der Gefahr wohl bewusst war, aber damit rechnete, dass er zurückkehren würde.

Was die Bewohner von Kanakia und von Aigina bewog, im Laufe des 12. Jhs. v. Chr. ihre Heimat zu verlassen, kann bestenfalls vermutet werden. Jedenfalls fällt diese Abwanderung in eine Zeit, die generell durch Veränderungen in den Siedlungsverhältnissen und durch Bevölkerungsfluktuationen gekennzeichnet war. Manche Regionen wurden verlassen, während anderswo neue Siedlungen gegründet wurden, und auch in der Belegung der Nekropolen spiegeln sich die Veränderungen in den Bevölkerungszahlen ${ }^{72}$. Was Salamis anlangt, so meint Y. Lolos, dass es zu einer Auswanderung nach Zypern kam. Diese These ist diskussionswürdig, doch sei darauf hingewiesen, dass bereits im 11. Jh. v. Chr. wieder neue Siedlungen und Friedhöfe im bergigen Inneren und im Ostteil von Salamis entstanden ${ }^{73}$. Aigina dagegen war im gesamten 11. Jh. v. Chr., also vom späten $\mathrm{SH}$ IIIC $^{74}$ über die submykenische und die frühe protogeometrische Periode bis ins 10. Jh. v. Chr. anscheinend verödet ${ }^{75}$. Auch das könnte darauf hindeuten, dass die Insel nicht erst um etwa 1120 verlassen wurde, sondern dass schon hunderte Jahre davor eine drastische Reduktion der Einwohnerzahlen stattgefunden hatte.

Y. Lolos interpretiert die Anlage, die er auf Kanakia ausgegraben hat, als den „Palast“ des Aias, der im Trojanischen Krieg die Kämpfer von Salamis anführte. Auch seine These von der Abwanderung der Salaminier nach Zypern kann sich auf den Mythos berufen, wenngleich in diesem Fall nicht Aias, sondern sein Halbbruder Teukros als Anführer agierte. Spinnt man jedoch den Mythos weiter, dann darf daran erinnert werden, dass Telamon, der Vater des Aias und des Teukros, in der Generation vor dem Trojanischen Krieg aus Aigina als Flüchtling nach Salamis gekommen $\operatorname{war}^{76}$.

69 Mountjoy 1999 (wie Anm. 3) 496.

70 Hiller 2003 (wie Anm. 6) 12 und Fig. 1 a, b: Krater aus SH IIIC Mitte/Fortgeschritten. - Hiller 2006 (wie Anm. 4) 77 und Tfl. II. E8: Skyphos aus SH IIIC Mitte.

7 I Lolos 2003 (wie Anm. 29) 81.

72 Überblick bei Deger-Jalkotzy wie Anm 53.

73 Vgl. Y. G. Lolos 2001 (wie Anm. 31).

74 Hiller 1975 (wie Anm. 2) Tfl. 38.374 präsentiert eine im British Museum aufbewahrte Bügelkanne aus SH IIIC Spät, angeblich ein Grabfund aus Aigina. Selbst wenn diese Zuweisung korrekt ist, wäre dieses Einzelstück nicht von großer Aussagekraft.

75 Hiller 2003 (wie Anm. 6).

76 Vgl. Diodor 4, 72, 7; Philostephanos, Kommentare fr. 35 (= FGrH III, 33). - Jörg Weilhartner sei für den Hinweis auf diese Stellen gedankt. 
Sigrid Deger-Jalkotzy

\section{Abbildungsnachweis:}

Abb. 1. Aigina-Kolonna. Abschnitt der Befestigungsanlage im Nordosten. Photoarchiv Grabung Aigina, Salzburg.

Abb. 2. Aigina-Kolonna. Abschnitt der Befestigungsanlage im Nordosten, Detail. Photoarchiv Grabung Aigina, Salzburg. Abb. 3. Salamis-Kanakia, Bau Gamma. Archiv Prof. Dr. Y. G. Lolos, Ioannina.

Abb. 4. Salamis-Kanakia, Korridorhäuser. Archiv Prof. Dr. Y. G. Lolos, Ioannina.

Abb. 5. Salamis-Kanakia, Bau Iota-Beta. Archiv Prof. Dr. Y. G. Lolos, Ioannina. 


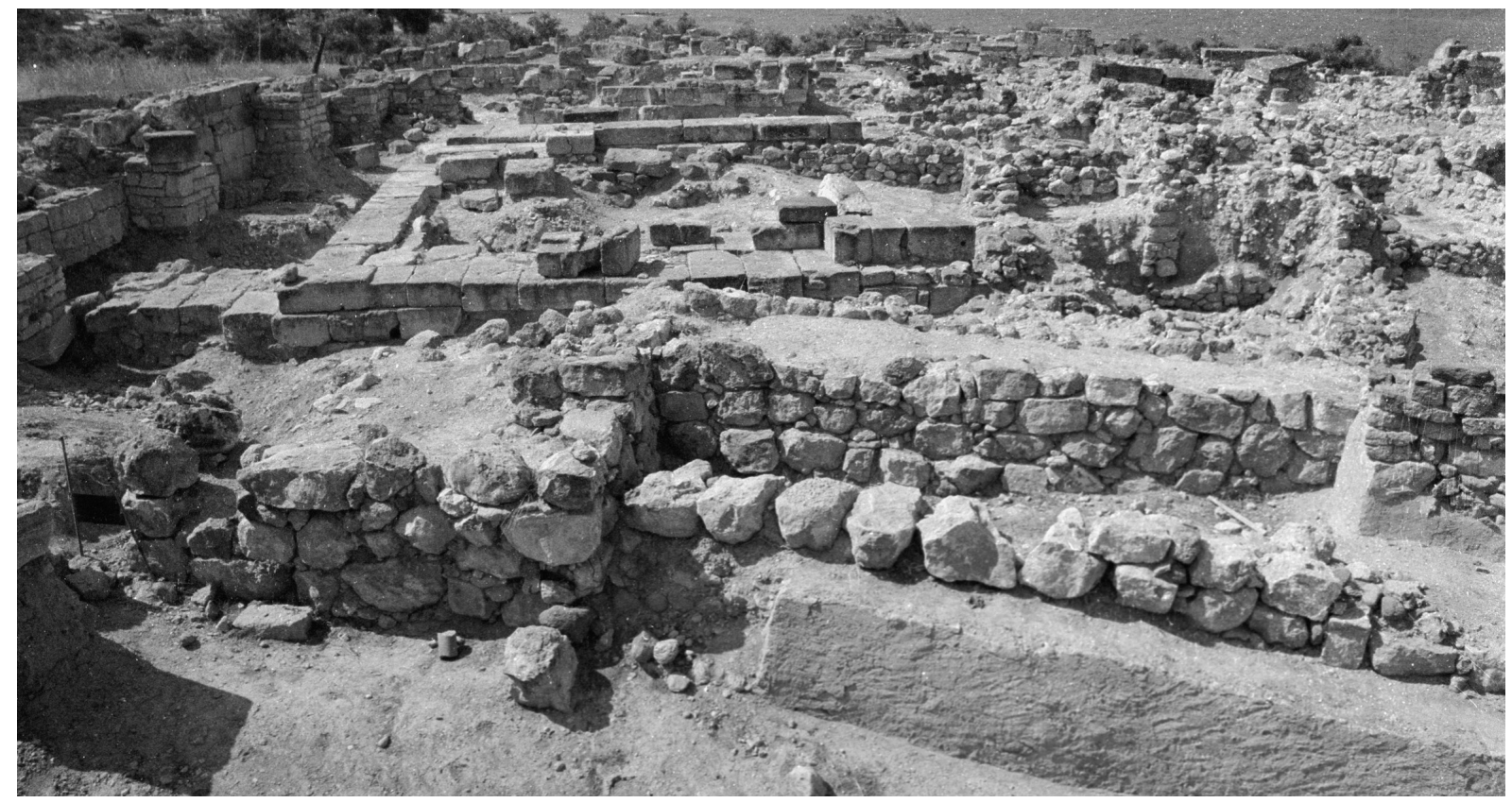

Abb. 1 Aigina-Kolonna. Abschnitt der Befestigungsanlage im Nordosten
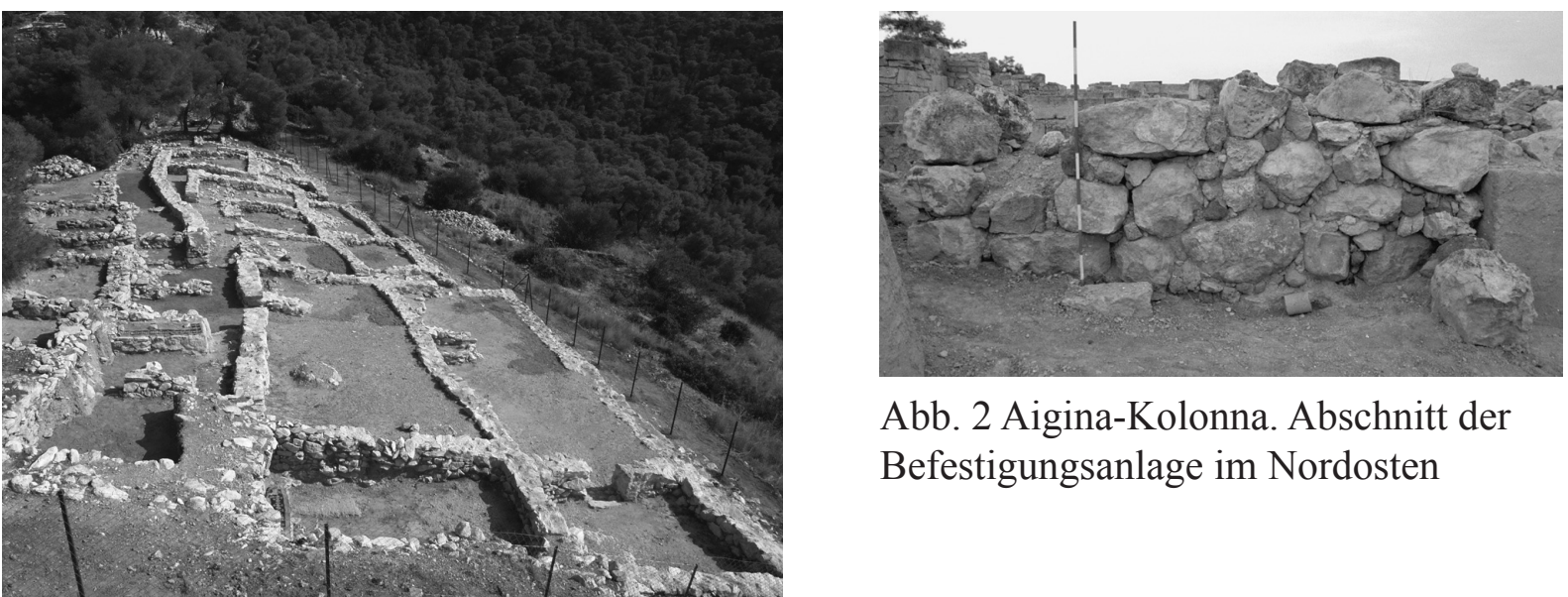

Abb. 2 Aigina-Kolonna. Abschnitt der Befestigungsanlage im Nordosten

Abb. 3 Salamis-Kanakia, Bau Gamma

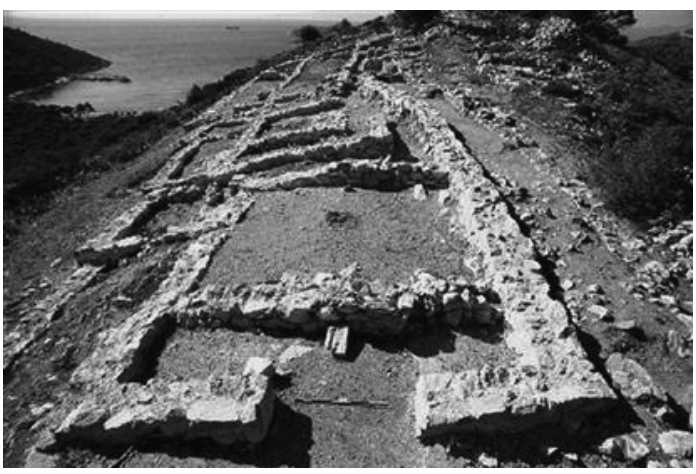

Abb. 4 Salamis-Kanakia, Korridorhäuser

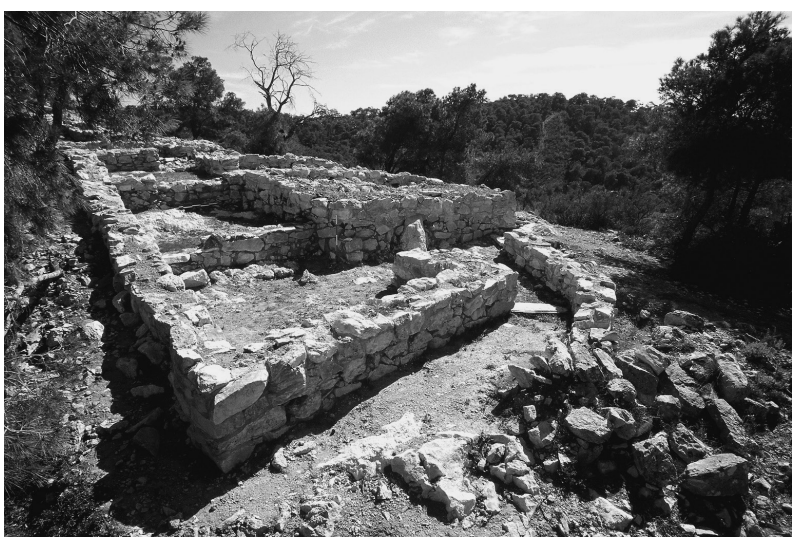

Abb. 5 Salamis-Kanakia, Bau Iota-Beta 